\title{
On the Coverage Extension and Capacity Enhancement of Inband Relay Deployments in LTE-Advanced Networks
}

\author{
Abdallah Bou Saleh, ${ }^{1,2}$ Simone Redana, ${ }^{1}$ Jyri Hämäläinen, ${ }^{2}$ and Bernhard Raaf ${ }^{1}$ \\ ${ }^{1}$ NSN Research - Radio Systems, Nokia Siemens Networks, St.-Martin-Strasse 76, 81541 Munich, Germany \\ ${ }^{2}$ Department of Communications and Networking, Aalto University School of Science and Technology, P.O. Box 3000, \\ Aalto, FIN-02015, Espoo, Finland
}

Correspondence should be addressed to Abdallah Bou Saleh, abdallah.bou_saleh.ext@nsn.com

Received 1 April 2010; Accepted 30 May 2010

Academic Editor: Wenbo Bo Wang

Copyright (c) 2010 Abdallah Bou Saleh et al. This is an open access article distributed under the Creative Commons Attribution License, which permits unrestricted use, distribution, and reproduction in any medium, provided the original work is properly cited.

Decode-and-forward relaying is a promising enhancement to existing radio access networks and is currently being standardized in 3GPP to be part of the LTE-Advanced release 10. Two inband operation modes of relay nodes are to be supported, namely Type 1 and Type $1 \mathrm{~b}$. Relay nodes promise to offer considerable gain for system capacity or coverage depending on the deployment prioritization. However, the performance of relays, as any other radio access point, significantly depends on the propagation characteristics of the deployment environment. Hence, in this paper, we investigate the performance of Type 1 and Type $1 \mathrm{~b}$ inband relaying within the LTE-Advanced framework in different propagation scenarios in terms of both coverage extension capabilities and capacity enhancements. A comparison between Type 1 and Type $1 \mathrm{~b}$ relay nodes is as well presented to study the effect of the relaying overhead on the system performance in inband relay node deployments. System level simulations show that Type 1 and Type $1 \mathrm{~b}$ inband relay deployments offer low to very high gains depending on the deployment environment. As well, it is shown that the effect of the relaying overhead is minimal on coverage extension whereas it is more evident on system throughput.

\section{Introduction}

The Universal Mobile Telecommunications System (UTR AN) Long Term Evolution (LTE) is being designed to enhance third generation (3G) radio access technologies (RATs) and it represents a major step towards the International Mobile Telecommunications- (IMTs-) Advanced technologies of the International Telecommunication UnionRadiocommunications Sector (ITU-R). The IMT-Advanced RATs are expected to offer increased broadband capacity with high quality of service (QoS) for next generation multimedia services, such as, high-definition TV (HDTV) content, video chat, mobile TV, and real-time gaming. Requirements for the IMT-Advanced technologies are defined by ITU-R in a circular letter issued in March 2008 calling for candidate RATs [1].

In response to ITU-R circular letter, 3rd Generation Partnership Project (3GPP) made a formal submission in September 2009 proposing that LTE Rel.10 and beyond would be evaluated as a candidate IMT-Advanced technology
[2]. The proposed RAT is referred to as LTE-Advanced and is expected to satisfy and overtake IMT-Advanced requirements [3]. In technology evolution, LTE-Advanced defines the framework for further significant advancements to LTE Rel.8 and Rel.9.

While LTE Rel.8 supports peak data rates exceeding $300 \mathrm{Mbps}$ in the downlink (DL) and $75 \mathrm{Mbps}$ in the uplink (UL), LTE-Advanced Rel.10 is expected to offer up to 1 Gbps in the DL and $500 \mathrm{Mbps}$ in the UL in low mobility environments. Extended carrier bandwidths up to $100 \mathrm{MHz}$ will be supported in LTE-Advanced while the maximum bandwidth in Rel. 8 is limited to $20 \mathrm{MHz}$. Furthermore, increased spectral efficiency up to $30 \mathrm{bps} / \mathrm{Hz}$ in $\mathrm{DL}$ and $15 \mathrm{bps} / \mathrm{Hz}$ in UL, along with improved cell edge capacity, decreased user and control plane latencies, and a more homogeneous user experience over the cell area are urged [3].

To address these stringent requirements, different key technologies have been investigated in the 3GPP study item 
on LTE-Advanced, of which bandwidth extension through spectrum/carrier aggregation, relay node (RN) deployments, improved multiple-input multiple-output (MIMO) schemes, coordinated multipoint transmission and reception (CoMP), and local area optimization features such as femtocell deployments $[4,5]$. In this paper, we will focus on relay nodes as a major technology enhancement.

Decode-and-forward (DF) relay nodes are currently being specified in the 3GPP work item on LTE-Advanced networks to meet the growing demand and challenging requirements for coverage extension and capacity enhancement [5]. RNs are characterized by wireless backhaul and low power consumption that is due to their relatively small size. The connection between $\mathrm{RN}$ and the core network is carried out through evolved Node B (eNB), referred to as the donor eNB in 3GPP terminology. The wireless backhaul enables deployment flexibility and eliminates the high costs of a fixed backhaul. Furthermore, RNs do not have strict installation guidelines with respect to radiation, visual disturbance, and planning regulation. Therefore, installing RNs involves lower operational expenditure (OPEX) [6] and faster network upgrade when operators aim to improve the QoS [7]. The cost efficiency of RNs is further investigated in $[6,8,9]$. Due to the compact physical characteristics and low power consumption, RNs can be mounted on structures like lamp posts with power supply facilities. According to previous technical studies, RNs promise to increase the network capacity $[10,11]$ and to better distribute resources in the cell, or alternatively, extend the cell coverage area [11$14]$.

In 3GPP standardization, relaying is being considered mainly as a cost-efficient coverage improvement technology. Different RN categories are specified according to the applied spectrum usage approaches on the wireless backhaul link between RN and its donor eNB. Inband relaying utilizes the same spectrum on the relay backhaul as that used to serve the user equipments (UEs) in the RN cells whereas outband RNs use a different spectrum. With the relay (eNB-RN) and access (RN-UE) links being time-division multiplexed in the former approach, operating both links on a single carrier frequency will impose limitations on the resource utilization efficiency, thus, limiting the performance of inband RNs. On the other hand, the latter approach relaxes the resource limitation on the relay link but it may increase the deployment costs since a separate extra spectrum is needed [5]. Considering the scarcity of available spectrums and the high costs of licensed ones, another possible mean to boost the performance of relays is to introduce enough isolation between the antennas used for the access and relay links, for example, provided by means of well-separated and well-isolated antenna structures. This will allow simultaneous operation of both links and, thus, relaxes the relay link.

In 3GPP, RNs are classified according to their relaying strategy. Three types of relays are identified out of which Type 1 and Type $1 \mathrm{~b}$ inband relays and Type 1a outband RNs are expected to be supported by LTE Rel.10 [5]. A Type $1 \mathrm{RN}$ controls a cell of its own, that is, it has its own physical cell ID and includes functionalities such as radio resource management, scheduling, and hybrid automatic repeat request (HARQ) retransmission control. Type 1a and Type $1 \mathrm{~b}$ RNs are characterized by the same set of features as Type $1 \mathrm{RN}$ above, except that the former utilizes outband backhaul spectrum usage approach, whereas the latter operates inband but with adequate antenna isolation between access and relay links.

In this paper, we investigate the performance of Type 1 and Type $1 \mathrm{~b}$ inband RNs in different propagation scenarios within the LTE-Advanced framework. Performance evaluation focuses on both coverage extension and network capacity aspects. In the former case, results are given in terms of an exchange ratio between the relay nodes and macro-cellular eNBs. That is, we examine how many small nodes like relays are needed to replace a conventional eNB, while keeping the same system performance in terms of $10 \%$-ile throughput cumulative distribution function CDF level. We recall the exchange ratio from [14] where it has been explained in more details. Average cell throughput and throughput CDF plots assuming a fixed coverage area are also presented.

The goal of the work is two-folded. On one hand, the relative gain of certain heterogeneous deployments (inband RNs) in comparison with homogeneous (eNBonly) deployments is investigated in different propagation scenarios. The study gives insights for relay deployments of LTE-Advanced networks in different environments. As the performance of a network depends significantly on the propagation conditions, it is essential to validate the deployment of RNs in different environments and give guidelines to the deployment costs and prospective gains. On the other hand, it is important as well to investigate the effect of the relaying overhead required for the inband relay link.

We use system level simulations to investigate the impact of the deployment scenario on the RN performance. Moreover, comparison between Type 1 and Type $1 \mathrm{~b}$ relays will be carried out and also some analytical considerations are used to characterize the impact of modeling. Simulation results have been produced using a tool that follows up-todate 3GPP models.

The rest of the paper is organized as follows. In Section 2, the applied evaluation methodology is explained. In Section 3, the propagation scenarios as well as the system model and simulation assumptions are given. In Section 4, performance evaluation and analysis are carried out. The paper is then concluded in Section 5.

\section{Evaluation Methodology}

The performance of Type 1 and Type $1 \mathrm{~b}$ relay deployments is evaluated in terms of network coverage and capacity. In the latter case, the average cell throughput is used as a performance measure, while in the former case, results are given in terms of the exchange ratio between RNs and macrocellular eNBs. This exchange ratio reflects the coverage gains due to RN deployments.

In the following, we first explain the approach used to model Type 1 and Type $1 \mathrm{~b}$ relaying on a system level 


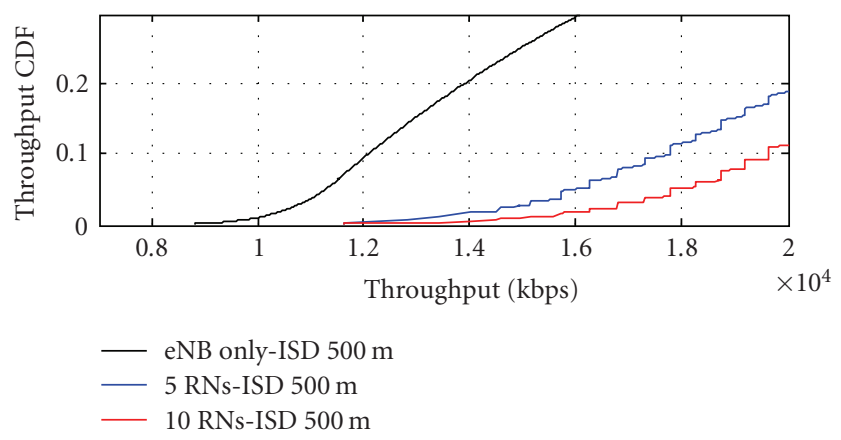

(a)

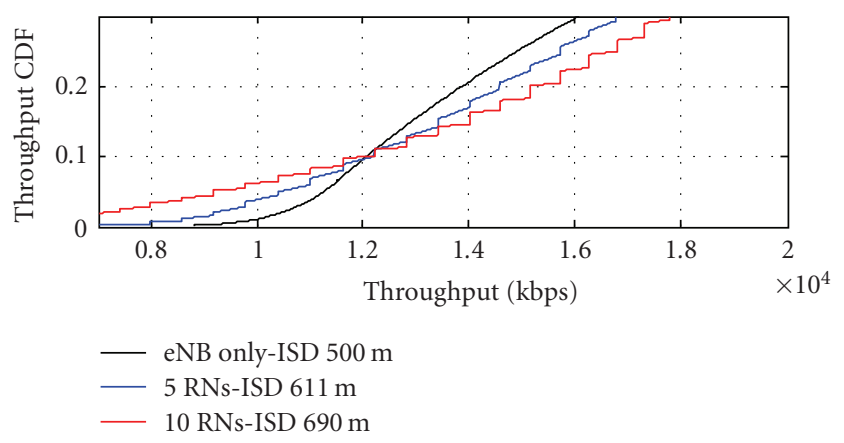

(b)

FigURE 1: Translating relay throughput gains into coverage extension.

performance evaluation. Then, we describe the methodology that is employed to quantify the coverage results.

2.1. Throughput Modeling for Type 1 and Type $1 b$ Relaying. The end-to-end (e2e) throughput experienced by a UE in a two-hop communication link (eNB-RN-UE) is given as follows:

$$
\mathrm{TP}_{\mathrm{e} 2 \mathrm{e}}=\min \left(\mathrm{TP}_{\mathrm{eNB}-\mathrm{RN}}, \mathrm{TP}_{\mathrm{RN}-\mathrm{UE}}\right),
$$

where e2e throughput is obtained as a minimum over throughputs on the relay and access links.

In inband relaying, the donor eNB utilizes the same timefrequency radio resource pool to serve both the RNs and UEs that are directly connected to it. As well, communications on the access and relay links are time-division multiplexed. Therefore, radio resources available for the relay link will be under high competition in the macrocell eNB. Since RN transmission power is low, its coverage area is relatively small when compared to the macrocell and a UE connected to an RN will usually experience a good access link. Thus, if UE rates are not limited in the $\mathrm{RN}$ cells, the relay link may become a bottleneck in two-hop e2e connections.

Throughout the study, we assume a resource allocation strategy which ensures equal data flows on the relay and access links. It is found from (1) that such an allocation is optimal because both relay and access links are fully utilized. Let $T_{x}$ be the portion of e2e connection resources available on link $x$, and let $R_{x}$ be the corresponding rate. Then, we have

$$
\begin{aligned}
\mathrm{TP}_{\mathrm{e} 2 \mathrm{e}}= & T_{\mathrm{eNB}-\mathrm{RN}} R_{\mathrm{eNB}-\mathrm{RN}}=T_{\mathrm{RN}-\mathrm{UE}} R_{\mathrm{RN}-\mathrm{UE}}, \\
& T_{\mathrm{eNB}-\mathrm{RN}}+T_{\mathrm{RN}-\mathrm{UE}}=1,
\end{aligned}
$$

where the latter equality is used to normalize the total transmission time on the relay and access links. After combining the equations in (2), we obtain the following formula for e2e throughput:

$$
\mathrm{TP}_{\mathrm{e} 2 \mathrm{e}}=\left(\frac{1}{\mathrm{TP}_{\mathrm{eNB}-\mathrm{RN}}}+\frac{1}{\mathrm{TP}_{\mathrm{RN}-\mathrm{UE}}}\right)^{-1} .
$$

Throughout the performance analysis, (3) will be used to model the e2e throughput of Type 1 inband RNs. We emphasize that (3) takes into account the throughput limitation on the relay link.
If enough isolation between the access and relay links can be obtained, both links can be operated simultaneously, thus easing the limitations on the relay link. In such a case, it is reasonable to assume that the relay link capacity is not a limiting factor and the e2e performance is merely constrained by the access link. We have adopted this assumption for Type $1 \mathrm{~b}$ inband relaying in order to find bounds for the performance difference with the Type 1 inband relaying approach. Thus, from this on, we assume for Type $1 \mathrm{~b}$ relaying that

$$
\mathrm{TP}_{\mathrm{e} 2 \mathrm{e}}=\mathrm{TP}_{\mathrm{RN}-\mathrm{UE}} .
$$

2.2. Tradeoff between Different Deployments. The modeling of the previous section will be used to make throughput comparison between Type 1 and Type $1 \mathrm{~b}$ inband relaying in the same network topology. While such a comparison is network capacity oriented, it is also of great value to carry out coverage-oriented performance comparison. For that purpose, we use the evaluation methodology of [14]. It aims to quantify the trade-off between numbers of relays and macrocell eNBs on the condition that the coverage requirement is fixed. In this paper, the cell coverage is defined in terms of the $10 \%$-ile throughput CDF level. The 10\%-ile level reflects the performance of the worst UEs, which might go easily into outage. In $3 \mathrm{GPP}$, the same approach is used, but performance is measured on the 5\%-ile level.

Let us describe the applied comparison methodology in the following. Assume a predefined inter-site-distance $\mathrm{ISD}_{0}$ between macrocell eNBs and assume that RNs are deployed at the edge of each sector. Then, the additional relays will increase the system throughput with respect to the reference eNB-only deployment. Yet, if the system is scaled by increasing the ISD, then the cell edge throughput can be decreased until the new deployment admit the same 10\%ile throughput as with the reference eNB-only deployment. In the above procedure, the number of deployed RNs per sector can be varied to obtain different ISD and RN density combinations that fulfill the coverage criterion (10\%-ile throughput CDF level). The explained approach is illustrated in Figure 1 which presents the throughput CDF for different deployments. The throughput gain from deploying $5 \mathrm{RNs}$ and 10 RNs per sector in the network where the original ISD is $500 \mathrm{~m}$ translates into ISD extensions of $111 \mathrm{~m}$ and $190 \mathrm{~m}$, 


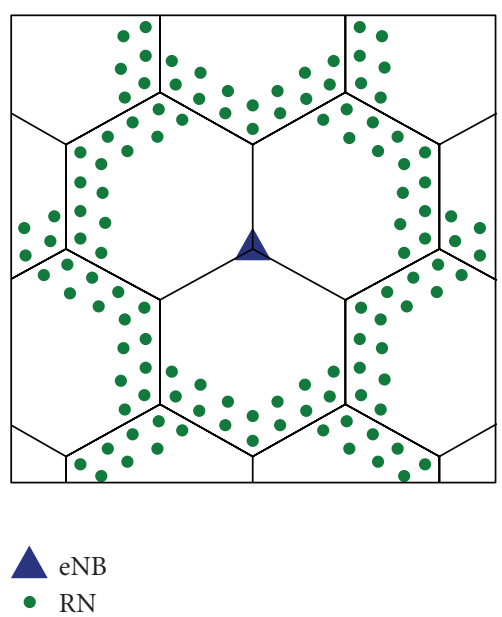

(a)

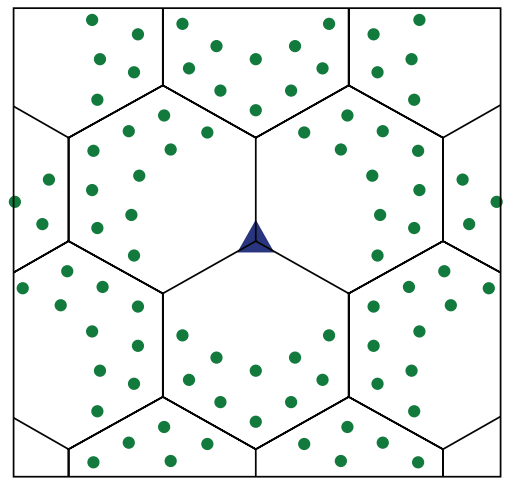

$\triangle \mathrm{eNB}$

- $\mathrm{RN}$

(b)

Figure 2: RN deployment in Scenario 1 (a) and Scenarios 2 and 3 (b).

respectively, while preserving the coverage performance at the 10\%-ile throughput level.

The different RN density and ISD combinations are referred to as ISO-performance deployments. The eNB-only deployment is referred to by the combination $\left(0, \mathrm{ISD}_{0}\right)$ and various $R N$ deployments are referred to by $\left(N_{\mathrm{RN}}, \mathrm{ISD}_{\text {ext }}\right)$, where $N_{\mathrm{RN}}$ refers to the number of RNs per sector. In the example shown in Figure 1, the combinations ( 0 RN, $500 \mathrm{~m})$, (5 RNs, $611 \mathrm{~m}$ ), and (10 RNs, $690 \mathrm{~m}$ ) are ISO-performance deployments.

The ISO-performance deployments are used to define the trade-off between numbers of RNs and eNBs. This tradeoff is expressed in terms of an exchange ratio that will be explained in the following. Assume a reference network that covers area $C$ and consists of $N_{0}$ macrocell sites, each composed by three sectors. If $N_{\mathrm{RN}}$ relays are added to each sector, starting from the outer edge of the sector (see Figure 2), then site coverage will be increasing and less sites will be needed in order to cover the area $C$. Let the number of sites in the RN deployment be $N_{\mathrm{eNB}}$. Then, the network contains $3 \cdot N_{\mathrm{RN}} \cdot N_{\mathrm{eNB}}$ relays, and we define the exchange ratio $R$ between RNs and macrocell eNBs by

$$
R=\frac{3 \cdot N_{\mathrm{RN}} \cdot N_{\mathrm{eNB}}}{N_{0}-N_{\mathrm{eNB}}} .
$$

Thus, the exchange ratio is defined by the number of RNs that are needed to reduce the number of macrocell sites from the original number $N_{0}$ down to $N_{\mathrm{eNB}}$. This ratio can be used to, for example, estimate the maximum costs of an $\mathrm{RN}$ site when the costs of macrocell sites are known. If, for example, $R=30$, then costs of an RN site can be at most $1 / 30$ of the costs of an eNB site.

Let us consider (5) more carefully. If performance evaluations are done by network level simulations, then it is usually not easy to change the number of sites in applied layout. Fortunately, the number of macrocell sites needed to cover a certain area is proportional to the square of ISD. Thus, there holds

$$
R=\frac{3 \cdot N_{\mathrm{RN}} \cdot N_{\mathrm{eNB}} / N_{0}}{1-N_{\mathrm{eNB}} / N_{0}}=\frac{3 \cdot N_{\mathrm{RN}} \cdot\left(\mathrm{ISD} / \mathrm{ISD}_{0}\right)^{2}}{1-\left(\mathrm{ISD} / \mathrm{ISD}_{0}\right)^{2}},
$$

and we can use a fixed number of macrocell sites since the performance gain will be obtained through the extended ISD between macrocell eNBs. We note that the ISD increase is limited by $10 \%$-tile throughput level constraint and multiple iterations of the network simulation might be needed before the proper ISD for a certain deployment is found.

It is worth noting that although the ISO-equivalent deployments perform similarly in terms of coverage, they may result in different exchange ratios according to the relative extension in ISD achieved by the number of RNs deployed. Then, the solution with the lowest cost is found by taking a minimum over the different achieved exchange ratios.

\section{Propagation Scenarios and System Model}

The performance of a mobile communication system depends largely on the radio environment and hence comparisons between different deployment alternatives and system level enhancements easily become unfair. Therefore, 3GPP has created guidelines for LTE-Advanced system evaluation methodology. The given simulation framework contains propagation and system models as well as recommended values for required parameters [5].

3.1. Propagation Models. Different environments exhibit different propagation characteristics which reflect on eNB and $\mathrm{RN}$ coverage areas rendering the network planning a rather challenging task. Small coverage areas may lead to high access node density and considerably high costs for operators. Hence, it is important to validate the RN deployments in different radio environments and give guidelines to the expected deployment costs. Due to increasing rate requirements, it is equally important to investigate the performance of RNs in terms of throughput in different propagation scenarios. 
It was early acknowledged in 3GPP LTE-Advanced study item that the propagation modeling is of essential importance when designing and assessing different RN deployments. This fact was reflected in the 3GPP discussion on the distance-dependent path loss model which was open for quite a long time during which the model was changed several times. The first proposed 3GPP model, given in [15], consists of only a non-line-of-sight (NLOS) component and is based on the NLOS ITU-R Urban Micro model [16]. The related scenario, which we will refer to as Scenario 1 (Sc1), assumes that both UEs and RNs always experience NLOS propagation conditions to their donor eNB and, thus, the socalled single slope model of the form

$$
\mathrm{PL}=\mathrm{PL}_{0}+10 \cdot n \cdot \log _{10}(R)
$$

is applied. In single slope models like Okumura-Hata, the constant term $\mathrm{PL}_{0}$ contains the impact of factors such as carrier frequency, and eNB and UE antenna heights, while the path loss exponent $n$ does not usually depend on the terminal antenna height. The model in (7) is feasible for densely built areas when a UE is on the street level and the line-of-sight (LOS) probability is small. In Table 1, parameters for different $3 \mathrm{GPP}$ path loss models are given [5]. It is seen that the difference between constant terms in the direct (eNB-UE) and relay links is $3.6 \mathrm{~dB}$. This is due to fact that $\mathrm{RN}$ antennas are expected to be elevated $5 \mathrm{~m}$ from ground level. The RN-UE path loss model shows more aggressive attenuation resulting from low $\mathrm{RN}$ antenna height.

The single slope model, however, is pessimistic since it does not take into account the fact that being in LOS conditions is becoming more and more probable when cell sizes are getting smaller. This is especially true when UEs are connected to RNs. Hence, the assumption of considering exclusively an NLOS connection as in [15] might be valid only in very densely populated cities. In the $3 \mathrm{GPP}$ evaluation framework, users are assumed to be indoors and the channel model is applied where the path loss towards the building is determined before adding the penetration loss. In many scenarios, there is an LOS connection or at least a clearly dominant direction in the channel between the $\mathrm{RN}$ and the building where the UE is located. Therefore, the link suffers from smaller path loss than the channel that assumes propagation over rooftops as in [16].

To address the above-explained propagation characteristics, a probabilistic dual slope model was proposed in [17] for RN-UE link. The model given in (8) is not a conventional dual slope model where a certain breakpoint distance is assumed; it considers the breakpoint through a probability and is based on measurements,

$$
\begin{gathered}
\mathrm{PL}=\operatorname{Prob}(\mathrm{LOS}) \cdot \operatorname{PL}(\mathrm{LOS})+\operatorname{Prob}(\mathrm{NLOS}) \cdot \mathrm{PL}(\mathrm{NLOS}) \\
\mathrm{PL}(\mathrm{LOS})=\mathrm{PL}_{\mathrm{LOS}}+10 \cdot n_{\mathrm{LOS}} \cdot \log _{10}(R) \\
\mathrm{PL}(\mathrm{NLOS})=\mathrm{PL}_{\mathrm{NLOS}}+10 \cdot n_{\mathrm{NLOS}} \cdot \log _{10}(R)
\end{gathered}
$$

The corresponding model, which will be referred to as Scenario 2 (Sc2) throughout this paper, assumes a mixed
LOS/NLOS modeling of the access channel; for parameters, see Table 1. The path loss on the access link is a weighted combination of two, LOS and NLOS, components, where the weighting factor decays as the UE-RN distance increases. The model in [17], however, does not consider environments, where users in a macrocell deployment may experience LOS propagation conditions with their donor eNB.

Finally, the 3GPP propagation Scenario 3 (Sc3), which is based on the model in [5], considers environments with better propagation conditions as compared to both models in Scenario 1 and Scenario 2. This scenario applies probabilistic dual slope model on all three links. It defines an LOS probability function versus the UE-eNB or UE-RN distance, and according to a random probability factor, the UE could be in LOS or NLOS propagation conditions. The model, thus, accounts for the case where UEs are in LOS condition with their eNB or RN, and as well for cases where a UE might be over-the-corner and, hence, might sometimes enjoy an LOS condition and in a nearby place an NLOS condition.

The scenarios reflect three different possible propagation conditions where relays can be deployed. Parameters of Table 1 have been accepted by 3GPP partners for performance evaluation of relay deployments in LTE-Advanced [5] and will be adopted throughout this paper.

3.2. System Model. The simulated network is represented by a regular hexagonal cellular layout with 19 trisectored macrocell sites. The RNs in each sector admit regular outdoor deployment at the sector border. Applied RN deployments will be discussed in Section 4 in more details. Simulation parameters follow the parameter settings agreed in 3GPP [5] and are summarized in Table 2. According to $3 \mathrm{GPP}$ performance evaluation guidelines, indoor users are assumed to be distributed with equal probability over the sector area and a $20 \mathrm{~dB}$ penetration loss is added on the access and direct links. The full-buffer traffic model is adopted. We note that due to outdoor RN deployment the penetration loss does not occur on the relay link (eNB-RN). Interference in the network is neglected, as the study investigates the performance of RNs in coveragelimited scenarios. Shadowing and fast fading are implicitly accounted for by a $30 \mathrm{~dB}$ extra margin on each link.

The link throughput in the system is calculated from signal to interference-plus-noise ratio (SINR) by using a mapping

$$
\mathrm{TP}=\mathrm{BW} \cdot B_{\mathrm{eff}} \cdot \log _{2}\left(1+\frac{\mathrm{SINR}}{\mathrm{SINR}_{\mathrm{eff}}}\right),
$$

where BW is the system operation bandwidth, and $B_{\text {eff }}$ and SINR $_{\text {eff }}$ are the so-called bandwidth and SINR efficiencies $[18,19]$. The approximation in (9) is obtained from the well-known Shannon link capacity after scaling by two parameters. This approximation method was introduced in [18], and it has been later used in 3GPP evaluations [19]. Bandwidth and SINR efficiencies depend on the antenna configuration, and we apply values which are given in Table 2. In simulations, we have used a $-7 \mathrm{~dB}$ limit on SINR so that a UE will be in outage if it experiences SINR levels less 
TAble 1: Propagation Models.

\begin{tabular}{|c|c|}
\hline \multicolumn{2}{|c|}{ Channel Models } \\
\hline Distance & $R[\mathrm{~km}]$ \\
\hline \multicolumn{2}{|c|}{ Scenario 1 (Sc1) } \\
\hline & eNB-UE Link \\
\hline & $\mathrm{PL}=128.1+37.6 \log _{10}(R)$ \\
\hline & RN-UE Link \\
\hline & $\mathrm{PL}=140.7+36.7 \log _{10}(R)$ \\
\hline & eNB-RN Link \\
\hline & $\mathrm{PL}=124.5+37.6 \log _{10}(R)$ \\
\hline \multicolumn{2}{|c|}{ Scenario 2 (Sc2) } \\
\hline & eNB-UE Link \\
\hline & $\mathrm{PL}=128.1+37.6 \log _{10}(R)$ \\
\hline & RN-UE Link \\
\hline & $\mathrm{PL}=\operatorname{Prob}(\mathrm{LOS}) \operatorname{PL}(\mathrm{LOS})+[1-\operatorname{Prob}(\mathrm{LOS})] \operatorname{PL}(\mathrm{NLOS})$ \\
\hline & PL(LOS): $103.8+20.9 \log _{10}(R)$, PL(NLOS): $145.4+37.5 \log _{10}(R)$ \\
\hline & ISD 500 m-Urban Model \\
\hline & $\operatorname{Prob}(\mathrm{LOS})=0.5-\min (0.5,5 \exp (-0.156 / R))$ \\
\hline & $+\min (0.5,5 \exp (-R / 0.03))$ \\
\hline & ISD 1732 m-Rural Model \\
\hline & $\operatorname{Prob}(\operatorname{LOS})=0.5-\min (0.5,3 \exp (-0.3 / R))$ \\
\hline & $+\min (0.5,3 \exp (-R / 0.095))$ \\
\hline & eNB-RN Link \\
\hline & $\mathrm{PL}=124.5+37.6 \log _{10}(R)$ \\
\hline \multicolumn{2}{|c|}{ Scenario 3 (Sc3) } \\
\hline & eNB-UE Link \\
\hline & PL(LOS): $103.4+24.2 \log _{10}(R), \operatorname{PL}($ NLOS $): 131.1+42.8 \log _{10}(R)$ \\
\hline & ISD $500 m$-Urban Model \\
\hline & $\operatorname{Prob}(\mathrm{LOS})=\min (0.018 / R, 1)(1-\exp (-R / 0.063))$ \\
\hline & $+\exp (-R / 0.063)$ \\
\hline & ISD 1732 m-Suburban Model \\
\hline & $\operatorname{Prob}($ LOS $)=\exp (-(R-0.01) / 0.2)$ \\
\hline & $R N$-UE Link \\
\hline & PL(LOS): $103.8+20.9 \log _{10}(R)$, PL(NLOS): $145.4+37.5 \log _{10}(R)$ \\
\hline & ISD 500 m-Urban Model \\
\hline & $\operatorname{Prob}(\mathrm{LOS})=0.5-\min (0.5,5 \exp (-0.156 / R))$ \\
\hline & $+\min (0.5,5 \exp (-R / 0.03))$ \\
\hline & ISD 1732 m-Suburban Model \\
\hline & $\operatorname{Prob}($ LOS $)=0.5-\min (0.5,3 \exp (-0.3 / R))$ \\
\hline & $+\min (0.5,3 \exp (-R / 0.095))$ \\
\hline & eNB-RN Link \\
\hline & PL(LOS): $100.7+23.5 \log _{10}(R)$, PL(NLOS): $125.2+36.3 \log _{10}(R)$ \\
\hline & ISD $500 m$-Urban Model \\
\hline & $\operatorname{Prob}(\mathrm{LOS})=\min (0.018 / R, 1)(1-\exp (-R / 0.072))$ \\
\hline & $+\exp (-R / 0.072)$ \\
\hline & ISD 1732 m-Suburban Model \\
\hline & $\operatorname{Prob}(\operatorname{LOS})=\exp (-(R-0.01) / 0.23)$ \\
\hline
\end{tabular}


TABle 2: Simulation Parameters.

\begin{tabular}{|c|c|}
\hline Parameter & $\begin{array}{l}\text { Value } \\
\text { Parameters }\end{array}$ \\
\hline Carrier Frequency & $2 \mathrm{GHz}$ \\
\hline Bandwidth & $10 \mathrm{MHz}$ \\
\hline Highest Modulation Scheme & 64-QAM $(R=9 / 10)$ \\
\hline Penetration Loss & $\begin{array}{l}20 \mathrm{~dB} \text { on eNB-UE and RN-UE } \\
\text { links }\end{array}$ \\
\hline Bandwidth Efficiency & 0.88 \\
\hline SINR Efficiency & 1.25 \\
\hline Thermal Noise PSD & $-174 \mathrm{dBm} / \mathrm{Hz}$ \\
\hline SINR lower bound & $-7 \mathrm{~dB}$ \\
\hline \multicolumn{2}{|c|}{ eNB Parameters } \\
\hline eNB Transmit Power & $46 \mathrm{dBm}$ \\
\hline eNB Elevation & $32 \mathrm{~m}$ \\
\hline eNB Antenna Gain & $14 \mathrm{dBi}$ \\
\hline eNB Antenna Configuration & $\mathrm{Tx}-2, \mathrm{Rx}-2$ \\
\hline eNB Noise Figure & $5 \mathrm{~dB}$ \\
\hline eNB Antenna Pattern & $A(\theta)=-\min \left[12\left(\theta / \theta_{3 \mathrm{~dB}}\right)^{2}, A_{m}\right]$ \\
\hline & $\Theta_{3 \mathrm{~dB}}=70^{\circ}$ and $A_{m}=25 \mathrm{~dB}$ \\
\hline \multicolumn{2}{|c|}{ UE Parameters } \\
\hline UE Antenna Configuration & $\mathrm{Tx}-1, \mathrm{Rx}-2$ \\
\hline UE Antenna Gain & $0 \mathrm{dBi}$ \\
\hline UE Height & $1.5 \mathrm{~m}$ \\
\hline UE Noise Figure & $9 \mathrm{~dB}$ \\
\hline \multicolumn{2}{|c|}{ Relay Node Parameters } \\
\hline RN Transmit Power & $30 \mathrm{dBm}$ \\
\hline RN Elevation & $5 \mathrm{~m}$ \\
\hline RN Antenna Configuration & $T x-2, R x-2$ \\
\hline RN-eNB Antenna Gain & $7 \mathrm{dBi}$ \\
\hline RN-UE Antenna Gain & $5 \mathrm{dBi}$ \\
\hline RN Antenna Pattern & Omni-directional \\
\hline RN Noise Figure & $5 \mathrm{~dB}$ \\
\hline
\end{tabular}

than $-7 \mathrm{~dB}$ and then the throughput is set to zero. This limit is introduced due to control channel requirements.

\section{Performance Evaluation and Analysis}

In this section, the performances of Type 1 and Type $1 \mathrm{~b}$ RNs are analyzed in terms of coverage extension and network capacity. First, we discuss RN deployments in the different propagation environments, then a bound on the number of RNs that can be deployed is deduced and, thereafter, we present the coverage extension and throughput results.

4.1. Relay Node Deployment. In LTE-Advanced, RNs will be deployed by operators in contrary to, for example, femtocells for which random deployment by end users may take place. Considering, as well, that RNs will be deployed to provide coverage improvements and a more homogeneous user experience over the cell area, we have systematically deployed RNs at the cell edge. Deployment is done in a way that no coverage gaps are left and overlapping between neighboring $\mathrm{RN}$ cells is minimized. The unnecessary holes in $\mathrm{RN}$ coverage would deteriorate the relative coverage extension gain from $\mathrm{RN}$ deployments, while the overlap between $\mathrm{RN}$ cells would lead to inefficient $\mathrm{RN}$ deployment.

The number of RNs required to cover the cell edge, however, depends significantly on the propagation environment since RNs will exhibit different coverage areas in different propagation conditions. Hence, careful planning of $\mathrm{RN}$ deployment is required.

Figure 2(a) presents RN deployments in Scenario 1, whereas RN deployments in Scenario 2 and Scenario 3 are shown in Figure 2(b). The deployments are nearly optimum in terms of the coverage improvement capabilities of the deployed RNs. In both Figures 2(a) and 2(b), 2 tiers of $\mathrm{RNs}$ are deployed. A tier of RNs is defined as the sufficient number of RNs required to cover the cell edge without introducing $\mathrm{RN}$ coverage gaps. In this study, $7 \mathrm{RNs}$ and 14 RNs, which constitute, respectively, 1 and 2 tiers, are deployed in Scenario 1 environments. The second tier is another set of RNs deployed closer to the eNB, however, still in a way where no RN coverage gaps are occurring between the two tiers. In Scenario 2 and Scenario 3, either 5 RNs or $10 \mathrm{RNs}$ are deployed. The different number of RNs per tier reflects the different coverage areas of RNs in different propagation environments. To keep the comparison fair, the same deployments are used for both Type 1 and Type $1 \mathrm{~b}$ inband RNs.

Tables 3 and 4 present, respectively, the RN coverage areas for the three considered scenarios in networks with ISD $500 \mathrm{~m}$ and ISD $1732 \mathrm{~m}$. We note that these ISD values have been widely used in 3GPP for the urban and suburban test cases. It is found that in case of $500 \mathrm{~m} \mathrm{ISD}$, the first tier of RNs covers about $20 \%$ of the macrocell sector area in Scenario 1 while in Scenario 2 the coverage is around $40 \%$. Similar behavior is experienced in $1732 \mathrm{~m}$ ISD case. We recall that the coverage area is defined by cell selection according to the received signal strength and, hence, the better propagation conditions on the access link in Scenario 2 due to the LOS component render observed larger coverage area. This conclusion is also valid for Scenario 3, but there, the potential LOS component occurs also in the direct link between $\mathrm{eNB}$ and UE and, therefore, RN coverage areas are somewhat smaller than in Scenario 2 for ISD $500 \mathrm{~m}$ case. In case of networks with ISD $1732 \mathrm{~m}$, however, Scenario 3 models a suburban environment where UEs towards the sector edge experience, with very high probability, NLOS propagation conditions to the eNB which are worse than those in Scenario 2. This leads to a larger coverage of RNs in Scenario 3 in ISD $1732 \mathrm{~m}$ case.

If two tiers of relays are employed, then the area covered by RNs is increasing, but we note that coverage cannot be doubled by doubling the number of relays. This is due to fact that $\mathrm{RN}$ coverage area will become smaller when it is moved closer to the eNB. We consider this phenomenon in the next subsection. 
4.2. Bound on the Number of Deployed Relays. From site coverage extension perspective, the outer edge of the macrocell sector offers the most attractive locations for relay deployments. Therefore, relays are usually deployed in tiers starting from the macrocell edge; see Figure 2, where RN deployments are illustrated. The number of relays in tiers is selected so that relays cover the whole macrocell edge. By adding new tiers between macrocell center and the first tier, the area covered by relays can be increased. Yet, the coverage gain from additional relay tiers will become smaller tier by tier. More specifically, while the relay link budget will define the maximum distance between the eNB and the first tier RNs, eNB interference on the access link may set a practical minimum distance between the eNB and RNs. To describe this in more details, we consider a user between the eNB and the $\mathrm{RN}$ in a location where the received powers from both are equal. Then,

$$
\frac{P_{\mathrm{eNB}}}{L_{\mathrm{eNB}-\mathrm{UE}}}=\frac{P_{\mathrm{RN}}}{L_{\mathrm{RN}-\mathrm{UE}}},
$$

where $P_{\mathrm{eNB}}$ and $P_{\mathrm{RN}}$ refer to the transmission powers of the eNB and the RN, respectively, and $L_{\mathrm{eNB}-\mathrm{UE}}$ and $L_{\mathrm{RN}-\mathrm{UE}}$ refer to the path losses on the links indicted by the subscript. Let us consider only distance-dependent path losses and antenna gains for simplicity. Then,

$$
\begin{gathered}
L_{\mathrm{eNB}-\mathrm{UE}}=G_{\mathrm{eNB}} \cdot \alpha_{\mathrm{eNB}} \cdot D_{\mathrm{eNB}}^{\beta_{\mathrm{NB}},} \\
L_{\mathrm{RN}-\mathrm{UE}}=G_{\mathrm{RN}} \cdot \alpha_{\mathrm{RN}} \cdot D_{\mathrm{RN}}^{\beta_{\mathrm{RN}}},
\end{gathered}
$$

where $D_{\mathrm{eNB}}$ and $D_{\mathrm{RN}}$ are distances from a UE to the eNB and the RN, respectively, $\left(\alpha_{\mathrm{eNB}}, \beta_{\mathrm{eNB}}\right)$ and $\left(\alpha_{\mathrm{RN}}, \beta_{\mathrm{RN}}\right)$ are path loss model parameters, and $G_{\mathrm{eNB}}$ and $G_{\mathrm{RN}}$ are the eNB and RN antenna gains, respectively. From (11) we solve

$$
D_{\mathrm{RN}}=D_{\mathrm{eNB}}^{\beta_{\mathrm{eNB}} / \beta_{\mathrm{RN}}} \cdot\left(\frac{\alpha_{\mathrm{eNB}}}{\alpha_{\mathrm{RN}}} \cdot \frac{G_{\mathrm{RN}}}{G_{\mathrm{eNB}}} \cdot \frac{P_{\mathrm{RN}}}{P_{\mathrm{eNB}}}\right)^{1 / \beta_{\mathrm{RN}}} .
$$

If we use parameters of Scenario 1 from Tables 1 and 2 and assuming that the $\mathrm{RN}$ is in the boresight direction from $\mathrm{eNB}$, then we obtain

$$
D_{\mathrm{RN}} \approx 0.095 \cdot D_{\mathrm{eNB}}^{1.02} .
$$

From the macrocell geometry, we find that if ISD is $500 \mathrm{~m}$, then the macrocell range is $333 \mathrm{~m}$. If $\mathrm{RN}$ is located close to the cell edge, then $D_{\mathrm{eNB}}$ is around $300 \mathrm{~m}$ since $D_{\mathrm{RN}}$ would be then about $28 \mathrm{~m}$. Thus, RN range is quite small even for first tier relays in Scenario 1. Furthermore, if RN is placed close to the midpoint between cell edge and eNB, and assume $D_{\mathrm{eNB}}=160 \mathrm{~m}$, for example, then we have $D_{\mathrm{RN}}=$ $15 \mathrm{~m}$, and RN coverage becomes truly small. Fortunately, the situation in Scenario 2 and Scenario 3 will be more favorable for relaying, see, for example, Tables 3 and 4 .

4.3. Coverage Extension: ISO-Performance. In what follows, the coverage extension capabilities of Type 1 and Type $1 b$ $\mathrm{RN}$ deployments are investigated in different scenarios in ISD $500 \mathrm{~m}$ and $1732 \mathrm{~m}$ networks, respectively.

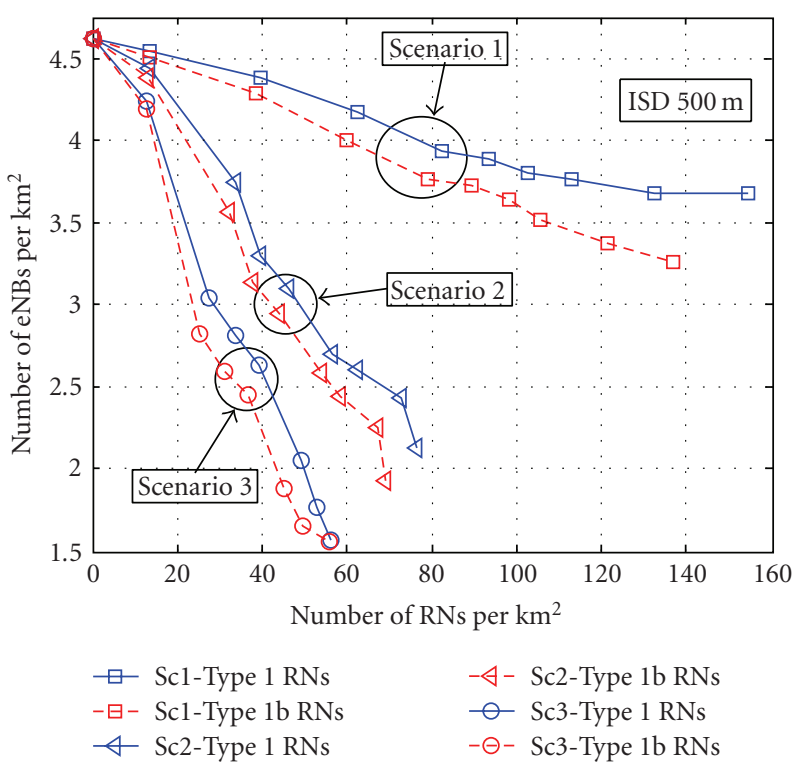

FIgURE 3: ISO-performance curve - ISD $500 \mathrm{~m}$.

TABle 3: RN Deployment Characteristics, ISD $500 \mathrm{~m}$.

\begin{tabular}{lccc}
\hline RN tiers per sector & Scenario & Number of RNs & $\begin{array}{c}\text { Total RN } \\
\text { coverage area } \\
{[\%]}\end{array}$ \\
\hline \multirow{3}{*}{1 tier } & Sc 1 & 7 & 19.5 \\
& Sc 2 & 5 & 39.5 \\
\multirow{3}{*}{2 tiers } & Sc 3 & 5 & 35.5 \\
& Sc 1 & 14 & 33 \\
& Sc 2 & 10 & 65 \\
\hline
\end{tabular}

TABLe 4: RN Deployment Characteristics, ISD $1732 \mathrm{~m}$.

\begin{tabular}{lccc}
\hline RN tiers per sector & Scenario & Number of RNs & $\begin{array}{c}\text { Total RN } \\
\text { coverage area } \\
{[\%]}\end{array}$ \\
\hline \multirow{3}{*}{1 tier } & Sc 1 & 7 & 21 \\
& Sc 2 & 5 & 39 \\
\hline \multirow{3}{*}{2 tiers } & Sc 3 & 5 & 48 \\
& Sc 1 & 14 & 36.5 \\
& Sc 2 & 10 & 61.5 \\
\hline
\end{tabular}

Figure 3 presents the ISO-performance of Type 1 and Type $1 \mathrm{~b}$ RNs for different scenarios in urban environments. Two conclusions are clearly deductible. On one hand, the ISO-performance differences between Type 1 and Type $1 \mathrm{~b}$ RNs are small in all three scenarios. In terms of coverage extension, the resources consumed by the relay link have limited effect on the system performance. On the second hand, there is a significant difference between the ISOperformances of $\mathrm{RN}$ deployments when using different path loss models. The required numbers of RNs in ISO 
combinations of Scenario 3 are less than those in Scenario 2 , which in turn is much more favorable for relaying than Scenario 1. When comparing Scenario 1 and Scenario 2, the better performance on the access link of Scenario 2 due to LOS probability clearly reflects on the performance of all UEs connected to RNs. We emphasize that the direct link path loss between eNB and UE as well as the path loss between eNB and RN are the same for Scenario 1 and Scenario 2. Thus, for both Type 1 and Type $1 b$ relays the difference between Scenario 1 and 2 ISO curves of Figure 3 is a direct consequence of the difference in the access link attenuation between RN and UE.

While comparing Scenario 2 and Scenario 3, it should be noted that the access link between RN and UE admits similar model in these scenarios while direct and relay link models are different. The better performance of Scenario 3 is mainly due to improved direct link. However, the LOS probability model in Scenario 3 significantly improves the performance of UEs close to the eNB, that is, those experiencing relatively good throughput levels anyway, and results in moderate performance improvement for the UEs at the edges between eNB and RN coverage areas. These are the UEs that contribute the most to the 10\%-ile throughput CDF level. On the contrary, as in Scenario 2, the access link model of Scenario 3 ensures significantly better UE throughput for users connected to RNs. Also, Type 1 inband relays can utilize the increased access link performance well in Scenario 3 since LOS probability is introduced to the relay link. Added to that, simulations show that UEs contributing to percentile levels higher than the 10\%-ile in throughput CDF are much better in Scenario 3 than those at the $10 \%-$ ile level when compared to the relative performance of both groups (UEs below 10\%-ile level and those above 10\%-ile level) in Scenario 2. Knowing that these UEs will eventually contribute to the $10 \%$-ile as the ISD increases, a higher margin of ISD extension can be supported in Scenario 3. These reasons lead to better coverage extension capabilities for RNs in Scenario 3 as compared to Scenario 2.

Table 5 presents the exchange ratio, calculated using (6), between the RNs and the macrocell eNB in the three considered scenarios. The values in Table 5 are selected based on the possible ISO-performance combinations so that the exchange ratio is minimized, that is, the selected $\left(N_{\mathrm{RN}}\right.$, ISD) combination is the one which achieves the largest ISD extension relative to the number of RNs and eNBs deployed per unit area. Simulations show that in Scenario 1, deploying 7 Type 1 RNs achieves an extension of $42 \mathrm{~m}$, whereas deploying 7 Type $1 \mathrm{~b}$ RNs results in a $54 \mathrm{~m}$ ISD extension. In Scenario 2, the best exchange ratio is achieved by deploying 4 or 7 Type 1 RNs, which achieves ISD extensions of $92 \mathrm{~m}$ and $154 \mathrm{~m}$, respectively. For Type $1 \mathrm{~b}$ RNs, 4-RN deployment is the most cost-efficient deployment and it results in an ISD extension of $107 \mathrm{~m}$. For Scenario 3, both Type 1 and Type $1 \mathrm{~b}$ RNs present the most cost-efficient ISO combination with 3 RNs per sector resulting in ISD extensions of $106 \mathrm{~m}$ and $140 \mathrm{~m}$, respectively.

The exchange ratios presented in Table 5 reflect the performances shown by the ISO plots of Figure 3 and values can be used to compare Type 1 and Type $1 \mathrm{~b}$ RNs in terms
TABLE 5: Coverage extension evaluation, ISD $500 \mathrm{~m}$.

\begin{tabular}{lcc}
\hline \multirow{2}{*}{ Considered Scenario } & \multicolumn{2}{c}{ Best exchange Ratio } \\
& Type 1 RN & Type 1b RN \\
\hline Scenario 1 & 120 & 93 \\
Scenario 2 & 30 & 26 \\
Scenario 3 & 18 & 15 \\
\hline
\end{tabular}

of costs. In Scenario 1, the deployment of Type 1 RNs is appealing if Type $1 \mathrm{RN}$ cost is lower than 93/120 times that of Type 1b RN. The cost should be lower than 26/30 and 15/18 times that of Type $1 \mathrm{~b}$ RNs in Scenario 2 and Scenario 3 , respectively. Note that the cost stands for the Total Cost of Ownership (TCO), and in such a case, the extra cost incurred to insure enough antenna isolation on the access and relay links in Type $1 \mathrm{~b}$ RN deployments should be taken into account.

Investigating the RN performance in the different scenarios, inband Type $1 \mathrm{RNs}$ are appealing, costwise, if the RN TCO is less than $1 / 120$ times that of a macro eNB. In Scenario 2 and Scenario 3, the cost limitation diminishes significantly down to $1 / 30$ and $1 / 18$ times that of an eNB. Similar behavior is noticed for Type $1 \mathrm{~b} \mathrm{RNs}$, where the cost-appealing ratio falls from $1 / 86$ in Scenario 1 to $1 / 26$ and 1/15 times the cost of an eNB in Scenario 2 and Scenario 3, respectively.

4.3.2. Rural/Suburban Models (ISD $1732 \mathrm{~m}$ ). Figure 4 shows the ISO-performance plots for Type 1 and Type $1 \mathrm{~b}$ RNs in different propagation conditions and ISD $1732 \mathrm{~m}$ network. Type 1 and Type $1 \mathrm{~b}$ RNs provide the same performance in terms of coverage extension, as they have overlapping ISO curves. This is an important indicator of the fact that the inband relay link for Type 1 relays does not incur noticeable costs on resource utilization provided that $\mathrm{RNs}$ are used to extend network coverage on rural/suburban areas. The exchange ratios between RNs and macro eNBs are presented in Table 6. Performance evaluation results of Figure 4 and Table 6 show a one-to-one exchange ratio between Type 1 and Type $1 \mathrm{~b}$ RNs.

An important result from the coverage extension study in both ISD $500 \mathrm{~m}$ and $1732 \mathrm{~m}$ cases is that the required number of Type $1 \mathrm{~b}$ relays is not much smaller than that of Type 1 relays. This is mainly due the fact that RNs are located outdoors, and even in Scenario 1 they admit a very good relay link towards the serving eNB. If network dimensioning is done assuming indoor users, then the radio resource usage by the backhaul of an outdoor Type $1 \mathrm{RN}$ is relatively small. Type 1 relay deployments, as compared to Type $1 \mathrm{~b}$ relay deployments, are hence well justified since they incur less costs than Type $1 \mathrm{~b}$ relays.

While comparing the system performances in different scenarios, similar behavior is visible as in the ISD $500 \mathrm{~m}$ network: The performance differs drastically between the scenarios. Exchange ratios vary from 86 RNs per eNB in Scenario 1 to $24 \mathrm{RNs}$ per eNB in Scenario 3. The exchange ratio for Scenario 1 is achieved by deploying 7 RNs, Type 1 or Type $1 \mathrm{~b}$, per sector, which results in a $201 \mathrm{~m}$ ISD extension. 
TABLE 6: Coverage extension evaluation, ISD $1732 \mathrm{~m}$.

\begin{tabular}{lcc}
\hline Considered Scenario & \multicolumn{2}{c}{ Best Exchange Ratio } \\
& Type 1 RN & Type 1b RN \\
\hline Scenario 1 & 86 & 86 \\
Scenario 2 & 28 & 28 \\
Scenario 3 & 24 & 24 \\
\hline
\end{tabular}

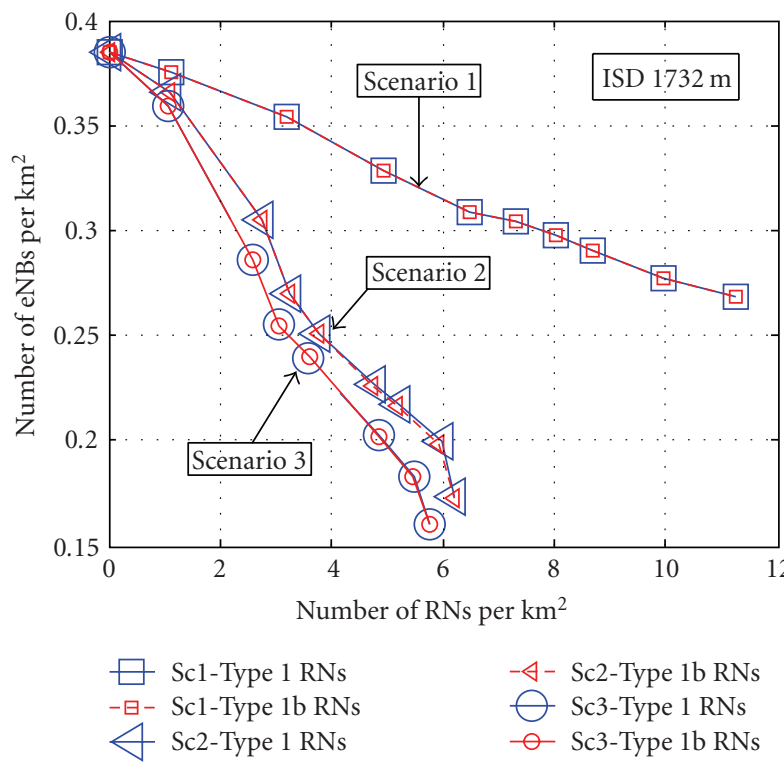

FIGURE 4: ISO-performance curve - ISD $1732 \mathrm{~m}$.

In Scenario 2, RN deployments with 5 RNs per sector achieve the best cost-efficient ISO-performance deployment and give an ISD extension of $414 \mathrm{~m}$. The exchange ratio in Scenario 3 is obtained by deploying 4 RNs which achieve an ISD extension of $396 \mathrm{~m}$. These performance differences among scenarios are due to applied path loss models and, therefore, the discussion and conclusions are the same as in case of ISD 500 m network.

Finally, we note that the exchange ratios were presented in connection with the corresponding $\mathrm{RN}$ deployments. If further coverage is required, a tradeoff between ISD extension and higher $\mathrm{RN}$ costs relative to the eNB may be considered.

4.4. Cell Throughput. In the following, the average cell throughput for the different scenarios will be presented for networks with ISD $500 \mathrm{~m}$ and $1732 \mathrm{~m}$, respectively. The performance of 1-Tier and 2-Tier Type 1 and Type $1 \mathrm{~b}$ relay deployments will be investigated and compared.

4.4.1. Urban Models (ISD $500 \mathrm{~m}$ ). Figure 5 presents the CDF of the cell throughput for Type 1 and Type $1 \mathrm{~b}$ relays assuming Scenario 1. It is clear that for both 7-RN and 14RN deployments, Type $1 \mathrm{~b}$ RNs outperform Type 1 RNs, specifically at the high throughput levels, where Type 1 RNs do not offer any throughput improvements over the macro eNB-only deployment. This is due to the relay link, which

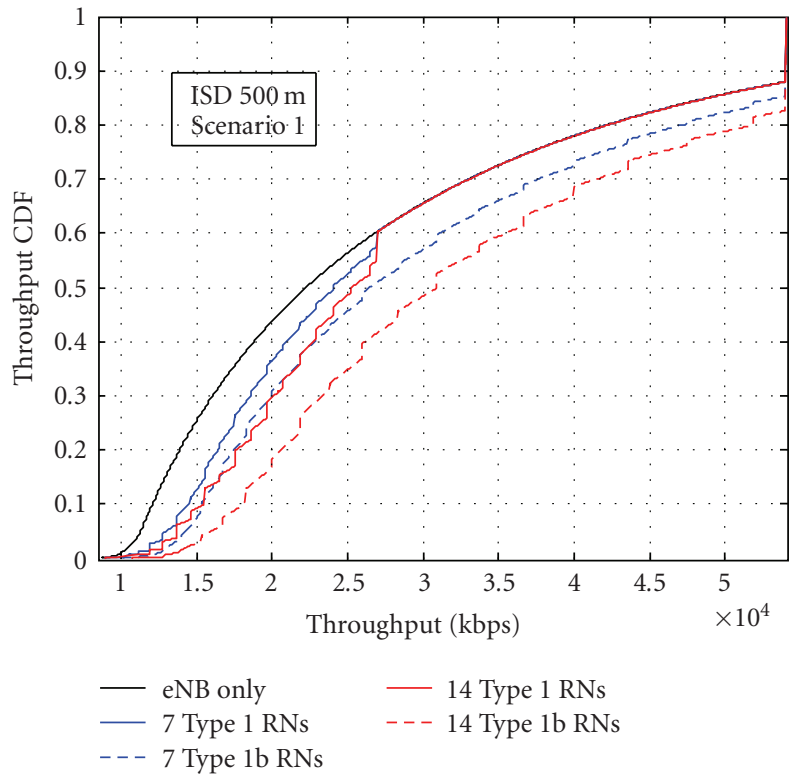

FIgURE 5: Throughput CDF-Scenario 1, ISD $500 \mathrm{~m}$.

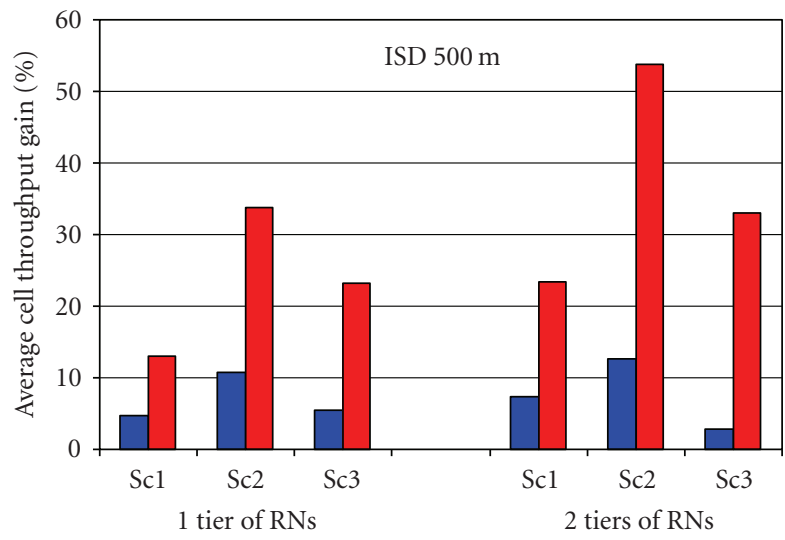

Type 1 RNs

Type 1b RNs

FIGURE 6: Average cell throughput gains in RN deployments, ISD $500 \mathrm{~m}$.

limits the e2e throughput over the two-hop link at high SINR levels to half of that that can be achieved on the direct link. This can be seen also from (2) and (3), where the e2e throughput of a UE served by a Type $1 \mathrm{RN}$ is formulated. Thus, only direct link users close to eNB contribute to the throughput CDF on very high throughput levels. Therefore, the peak rate limitation in $\mathrm{RN}$ cells is best observed from the step in the CDF plots of Figure 5 at $27 \mathrm{Mbps}$, where the performance of Type $1 \mathrm{RN}$ deployments converges to that of the eNB-only.

According to simulations, a 7-RN deployment with Type 1 relays provides 20\% improvement over the eNB-only deployment at the $10 \%$-ile throughput CDF level, whereas Type $1 \mathrm{~b}$ relays provide $27 \%$ gain. The observed gains at the 50\%-ile level are $8 \%$ and $18 \%$ from Type 1 and Type 
$1 \mathrm{~b} \mathrm{RN}$ deployments, respectively. The clear trend is that gains from Type $1 \mathrm{~b}$ relays against Type 1 relays become larger with increased throughput CDF levels. This is due to the fact that the relay link creates a bottleneck limiting the e2e throughput when access link radio conditions become better. The conclusion is that UEs connected to Type 1 RNs can in general experience better throughput than in eNB-only deployment, but only Type $1 \mathrm{~b}$ relaying clearly increases the number of users that admit extremely high throughput. The latter conclusion is backed up by Figure 6 that presents the achieved average cell throughput gains with respect to the eNB-only reference case. We note that 2tier deployment can be used to increase the cell rate when employing Type $1 \mathrm{~b}$ relays, but in case of Type 1 relays the gain from the second tier is small. It is worth noting that in the coverage investigations the large throughput performance difference between Type 1 and Type $1 \mathrm{~b}$ was not well visible in the coverage extension capabilities of relays because the difference at the $10 \%$-ile throughput level is small.

Since results of Figures 5 and 6 assume Scenario 1, it is worth to briefly discuss about the throughput in other scenarios. After comparing the performance of RNs in all three scenarios, we notice that relaying benefits can significantly differ, as was the case in the coverage extension study. RN deployments in Scenario 2 show better performance than in Scenario 1; introducing LOS component to the path loss model of the access link improves the performance of relay deployments whereas the eNB-only performance does not change. When comparing Scenario 2 and Scenario 3, it was found that $\mathrm{RN}$ deployments in the former scenario achieve higher relative gain in average cell throughput compared to the eNB-only deployment. This is due to the considerably high throughput levels of eNB-only deployments in Scenario 3 where the UEs close to the eNB experience an LOS connection and hence achieve much higher throughputs as compared to those in Scenario 2. Such UEs contribute significantly to the average cell throughput as compared to those on the cell edge. With the RN cells providing slightly better capacity improvements in Scenario 3 when compared to those in Scenario 2, the relative capacity improvement by $\mathrm{RNs}$ in the former is then smaller.

4.4.2. Rural/Suburban Models (ISD $1732 \mathrm{~m}$ ). Figure 7 presents the throughput CDF plots of 1-tier and 2-tier RN deployments assuming Scenario 3 propagation model. It is clearly observed that Type 1 and Type $1 \mathrm{~b}$ RNs show the same performance at the low percentile throughput CDF levels.

This confirms the previous result from the coverage extension study; resources consumed for the inband relay link in Type $1 \mathrm{RN}$ deployments do not impact the performance of cell edge UEs.

As in networks where ISD $=500 \mathrm{~m}$, Type $1 \mathrm{~b}$ RNs outperform Type 1 RNs at mid to high throughput levels where the relay link becomes a limiting factor as it requires large portion of the resources. It is, however, worth noting that the relative gain from Type $1 \mathrm{~b}$ RNs as compared to Type $1 \mathrm{RNs}$ is small.

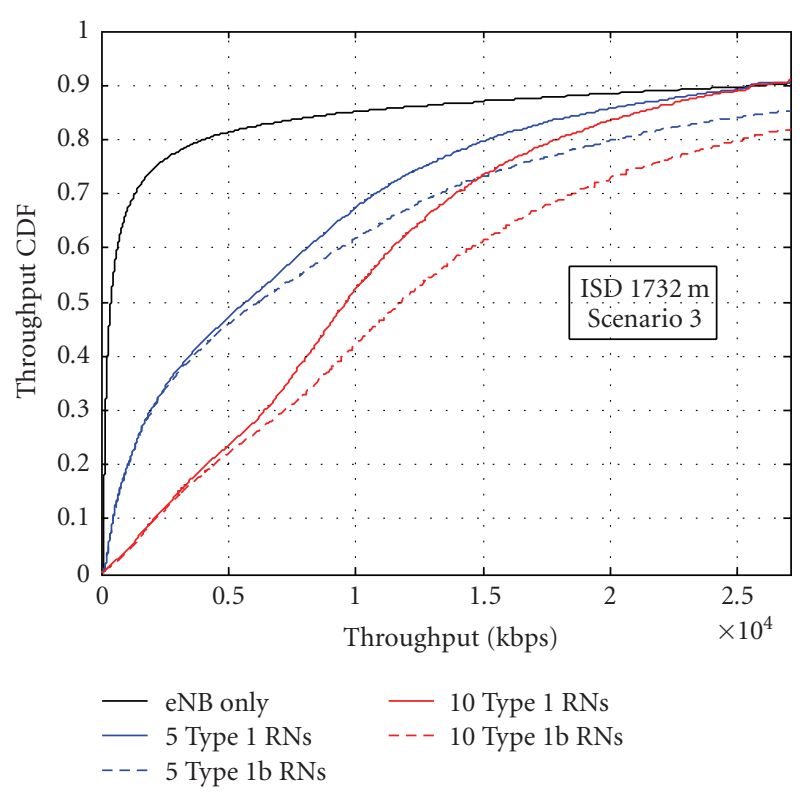

FIgURE 7: Throughput CDF-Scenario 3, ISD $1732 \mathrm{~m}$.

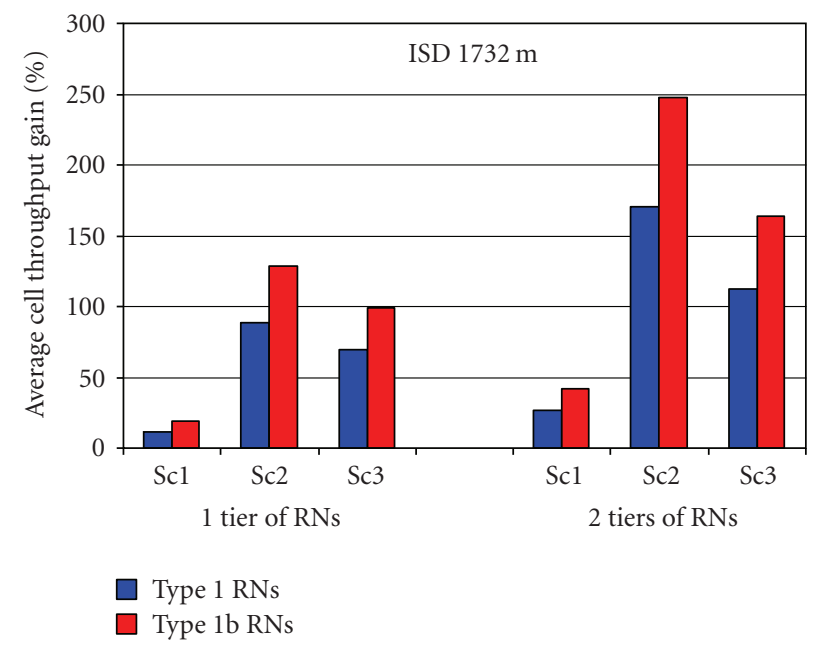

FIGURE 8: Average cell throughput gains in RN deployments, ISD $1732 \mathrm{~m}$.

This is observed in Figure 8, where the gains in average cell throughput are presented. It is clearly observed that gains from both 1-Tier and 2-Tier RN deployments are similar in case of Type 1 and Type 1b RNs. However, it is worth noting the significant difference in RN performance in the three scenarios. RN deployments perform remarkably better in Scenario 2 and Scenario 3 as compared to Scenario 1. This is due to the severe propagation loss on the access link in Scenario 1 where all UEs are assumed to be in NLOS conditions with the donor RN. Comparing Scenario 2 and Scenario 3, similar conclusions and justifications still hold as in networks with ISD $500 \mathrm{~m}$.

Note that significant capacity improvements are achieved by RN deployments in all scenarios as compared to eNB-only deployments. 


\section{Conclusion}

We compared the performance and feasibility of Type 1 and Type 1b Relay Node (RN) deployments within the LTEAdvanced framework. As performance measures, we used the cell coverage, that is, the exchange ratio between numbers of relays and evolved Node Bs (eNBs), and cell throughput. The evaluation was carried out for three different 3GPP propagation models in networks with inter-site-distances (ISDs) $500 \mathrm{~m}$ and $1732 \mathrm{~m}$.

First, it was analytically shown that the number of relays within a macrocell admits a practical upper bound since a relay coverage area decreases when it is moved closer to the eNB. Yet, it was noticed that the RN coverage depends largely on the applied path loss scenario. This has been also acknowledged by 3GPP where three different path loss scenarios have been introduced for LTE-Advanced performance evaluations. It was shown through system level simulations that relay deployments exhibit significantly different performance in the different scenarios in terms of both coverage and capacity-oriented evaluation criteria. RNs provided significant gains in scenarios where users exhibit good propagation conditions to the $\mathrm{RN}$, whereas modest gains were achieved in other scenarios.

As the relaying overhead in case of Type 1 relays was expected to be the limiting factor, we investigated the effect of inband relay backhaul link in the three 3GPPoriginated scenarios. Results show that the difference in coverage performance between Type 1 and Type $1 \mathrm{~b}$ relay deployments is small making the Type $1 \mathrm{RN}$ deployment attractive, especially in the case where network dimensioning assumes indoor users and outdoor relays. Actually, in suburban networks with ISD $1732 \mathrm{~m}$, Type 1 and Type $1 \mathrm{~b}$ $\mathrm{RN}$ deployments provide equal coverage performance. If ISD $500 \mathrm{~m}$ urban Scenario 1 is assumed, then Type $1 \mathrm{~b}$ relays provide slightly better coverage performance than Type 1 relays. Yet, it should be noted that 3GPP path loss Scenario 1 admits extremely aggressive signal attenuation between RN and UE making the use of small outdoor cells less favorable in general.

The investigations have shown that the capacity performance of Type 1 and Type $1 \mathrm{~b}$ inband relays differs significantly. Especially in ISD 500 m network, Type 1 relays clearly lag behind Type $1 \mathrm{~b}$ relays in throughput performance. The throughput difference is largest in high-throughput regimes.

\section{References}

[1] Circular Letter 5/LCCE/2, "Invitations for Submission of Proposals for Candidate Radio Interface Technologies for the Terrestrial Components of the Radio Interface(s) for IMTAdvanced and Invitation to Participating in Their Subsequent Evaluation," March 2008, http://www.itu.int/.

[2] 3GPP RP-090939, "3GPP Submission Package for IMTAdvanced," http://www.3gpp.org/.

[3] TR36.913, "Requirements for Further Advancements for Evolved Universal Terrestrial Radio Access (E-UTRA)," v9.0.0, December 2009, http://www.3gpp.org/.

[4] P. E. Mogensen, T. Koivisto, K. I. Pedersen et al., "LTEadvanced: the path towards gigabit/s in wireless mobile communications," in Proceedings of the 1st International Conference on Wireless Communication, Vehicular Technology, Information Theory and Aerospace and Electronic Systems Technology (VITAE'09), pp. 147-151, Aalborg, Denmark, May 2009.

[5] TR 36.814, "Further Advancements for E-UTRA: Physical Layer Aspects (Release 9)," v2.0.0, March 2010, http://www .3gpp.org/.

[6] E. Lang, S. Redana, and B. Raaf, "Business impact of relay deployment for coverage extension in 3GPP LTE-advanced," in Proceedings of IEEE International Conference on Communications Workshops (ICC '09), Dresden, Germany, June 2009.

[7] K. Doppler, S. Redana, M. Wódczak, P. Rost, and R. Wichman, "Dynamic resource assignment and cooperative relaying in cellular networks: concept and performance assessment," EURASIP Journal on Wireless Communications and Networking, vol. 2009, Article ID 475281, 14 pages, 2009.

[8] P. Moberg, P. Skillermark, N. Johansson, and A. Furuskär, "Performance and cost evaluation of fixed relay nodes in future wide area cellular networks," in Proceedings of IEEE International Symposium on Personal, Indoor and Mobile Radio Communications (PIMRC '07), pp. 1-5, Athens, Greece, September 2007.

[9] B. Timuş, "Cost analysis issues in a wireless multihop architecture with fixed relays," in Proceedings of the 61st IEEE Vehicular Technology Conference (VTC '05), pp. 3178-3182, Stockholm, Sweden, May-June 2005.

[10] A. So and B. Liang, "Effect of relaying on capacity improvement in wireless local area networks," in Proceedings of IEEE Wireless Communications and Networking Conference (WCNC '05), pp. 1539-1544, New Orleans, La, USA, March 2005.

[11] A. Bou Saleh, S. Redana, B. Raaf, and J. Hämäläinen, "Comparison of relay and pico eNB deployments in LTE-advanced," in Proceedings of the 70th IEEE Vehicular Technology Conference (VTC '09), Anchorage, Alaska, USA, September 2009.

[12] R. Schoenen, W. Zirwas, and B. H. Walke, "Capacity and coverage analysis of a 3GPP-LTE multihop deployment scenario," in Proceedings of IEEE International Conference on Communications Workshops, pp. 31-36, Beijing, China, May 2008.

[13] R. Schoenen, R. Halfmann, and B. H. Walke, "An FDD multihop cellular network for 3 GPP-LTE," in Proceedings of the 67th IEEE Vehicular Technology Conference (VTC '08), pp. 19901994, Marina Bay, Singapore, May 2008.

[14] T. Beniero, S. Redana, J. Hämäläinen, and B. Raaf, "Effect of relaying on coverage in 3GPP LTE-advanced," in Proceedings of the 69th IEEE Vehicular Technology Conference (VTC '09), Barcelona, Spain, April 2009.

[15] TR 36.814, "Further Advancements for E-UTRA: Physical Layer Aspects (Release 9)," v1.0.0, Januaray 2009, http://www .3gpp.org/.

[16] "Guidelines for Evaluation of Radio Interface Technologies for IMT-Advanced," Document 5D/TEMP/99 (Rev.1), http://www.itu.int/.

[17] TR 36.814, "Further Advancements for E-UTRA: Physical Layer Aspects (Release 9)," v1.0.1, March 2009, http://www .3gpp.org/.

[18] P. Mogensen, W. Na, I. Z. Kováes et al., "LTE capacity compared to the shannon bound," in Proceedings of the 65th IEEE Vehicular Technology Conference (VTC '07), pp. 1234-1238, Dublin, Ireland, April 2007.

[19] H. Holma and A. Toskala, LTE for UMTS, John Wiley \& Sons, New York, NY, USA, 2009. 

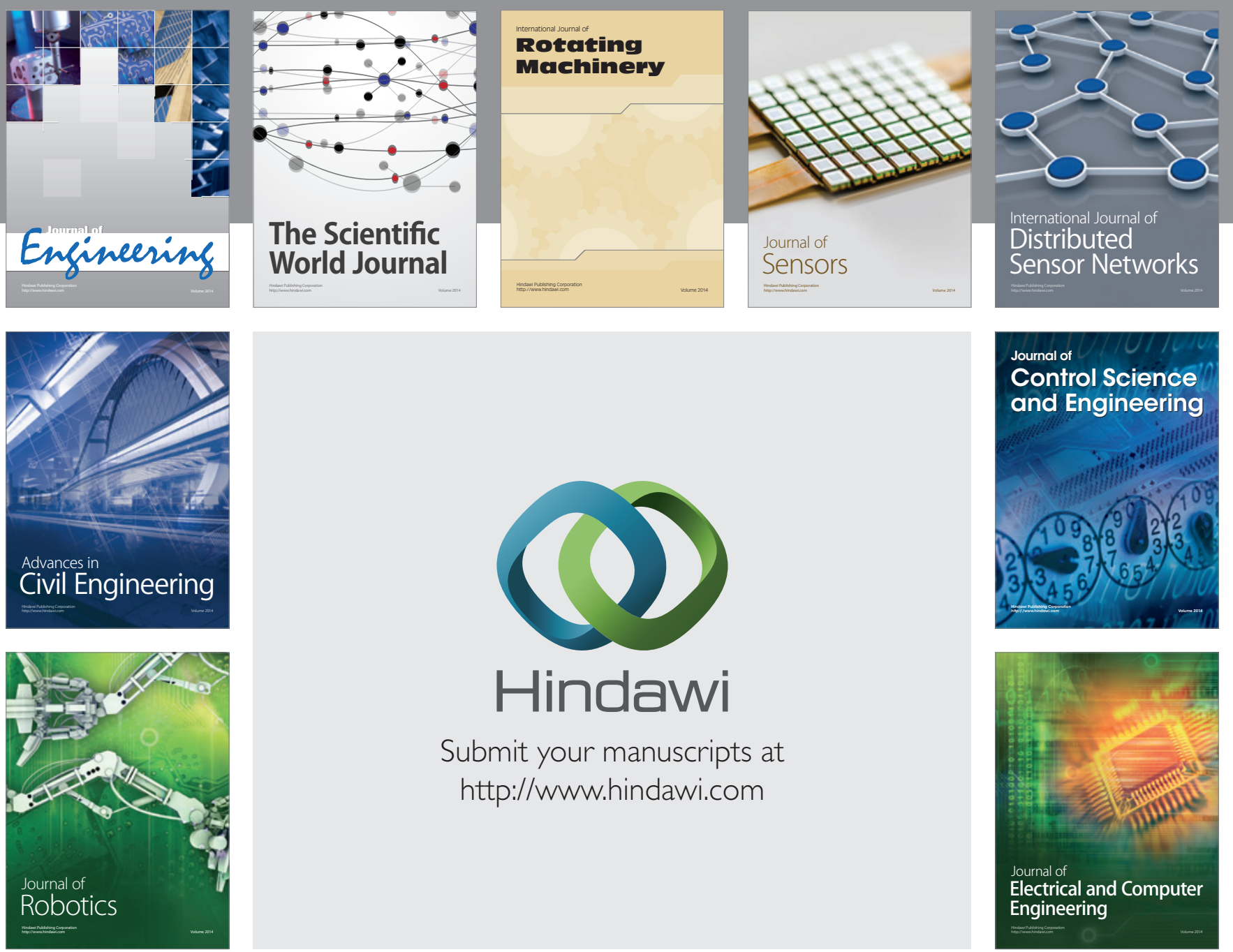

Submit your manuscripts at

http://www.hindawi.com
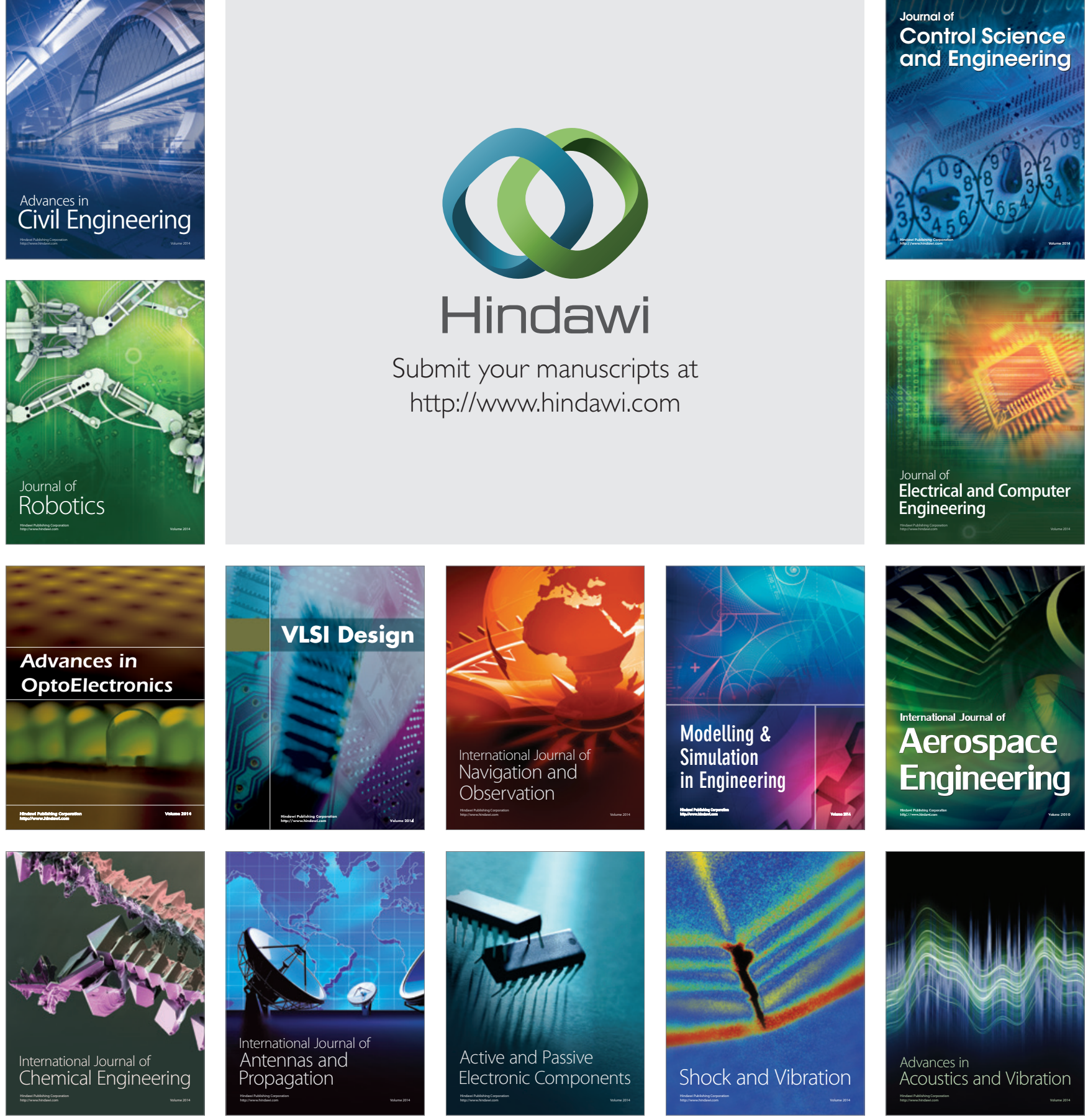